\title{
Perfectly-Secure MPC with Linear Communication Complexity ${ }^{\star}$
}

\author{
Zuzana Beerliová-Trubíniová and Martin Hirt \\ ETH Zurich, Department of Computer Science, CH-8092 Zurich \\ \{bzuzana, hirt\}@inf .ethz.ch
}

\begin{abstract}
Secure multi-party computation (MPC) allows a set of $n$ players to securely compute an agreed function, even when up to $t$ players are under the control of an adversary. Known perfectly secure MPC protocols require communication of at least $\Omega\left(n^{3}\right)$ field elements per multiplication, whereas cryptographic or unconditional security is possible with communication linear in the number of players. We present a perfectly secure MPC protocol communicating $\mathcal{O}(n)$ field elements per multiplication. Our protocol provides perfect security against an active, adaptive adversary corrupting $t<n / 3$ players, which is optimal. Thus our protocol improves the security of the most efficient information-theoretically secure protocol at no extra costs, respectively improves the efficiency of perfectly secure MPC protocols by a factor of $\Omega\left(n^{2}\right)$. To achieve this, we introduce a novel technique - constructing detectable protocols with the help of so-called hyper-invertible matrices, which we believe to be of independent interest. Hyper-invertible matrices allow (among other things) to perform efficient correctness checks of many instances in parallel, which was until now possible only if error-probability was allowed.
\end{abstract}

Keywords: Multi-party computation, efficiency, perfect security, hyperinvertible matrix.

\section{Introduction}

\subsection{Secure Multi-party Computation}

Secure multi-party computation (MPC) enables a set of $n$ players to securely evaluate an agreed function even when $t$ of the players are corrupted by a central adversary. A passive adversary can read the internal state of the corrupted players, trying to obtain some information he is not entitled to. An active adversary can additionally make the corrupted players deviate from the protocol, trying to falsify the outcome of the computation. In this work, we consider active adversaries.

The MPC problem dates back to Yao Yao82. The first generic solutions presented in GMW87, CDvdG87, GHY87 (based on cryptographic intractability assumptions) and later BGW88, CCD88, RB89, Bea91b (with informationtheoretic security) are rather inefficient and thus of theoretical interest mainly.

\footnotetext{
* This work was partially supported by the Zurich Information Security Center. It represents the views of the authors.
} 


\subsection{Efficiency of MPC Protocols}

In the recent years lots of research concentrated on designing protocols with lower communication complexity. In this paper we concentrate on bit-complexity, measured in bits sent by honest players. The following table gives an overview on the currently most efficient MPC protocols (in the respective security model), where $\kappa$ denotes the bit-length of a field element (resp. the security parameter).

\begin{tabular}{c|l|c|l} 
Thresh. & Security & Bits/Mult. & Reference \\
\hline$t<n / 3$ & perfect & $\mathcal{O}\left(n^{3} \kappa\right)$ & HMP00 \\
$t<n / 2$ & unconditional & $\mathcal{O}\left(n^{2} \kappa\right)$ & BH06 \\
$t<n / 2$ & cryptographic & $\mathcal{O}(n \kappa)$ & HN06 \\
$t<n / 3$ & unconditional & $\mathcal{O}(n \kappa)$ & DN07
\end{tabular}

All above protocols use "player elimination" (or its generalization "dispute control") - a technique that enables converting non-robust (but detectable) protocols into robust protocols, essentially without any efficiency loss. Furthermore, all but the perfectly secure protocol use circuit randomization Bea91a, which reduces the multiplication of two shared values to two reconstructions, given a precomputed sharing of a random multiplication triple $(a, b, c)$ with $c=a b$. Such triples can be non-robustly generated and checked in advance - making use of parallelization. Checking the correctness of many instances in parallel can be done very efficiently when negligible error-probability is allowed, however until now no perfectly secure efficient parallel correctness-checks are known.

\subsection{Contributions}

In this paper, we present a novel technique which, at the same time, allows to perfectly and very efficiently verify a bunch of sharings and (if the check says that they are correct) to extract a set of (new) correct random sharings given that a sub-set of the original sharings is random.

More precisely, given $n$ supposedly random sharings, up to $t$ of them distributed by corrupted players (and thus possibly of a wrong degree, non-random, etc), we can check whether they are all correct and if so (locally) compute $n-2 t$ correct and uniform random sharings. The check is (despite of being perfectly secure) highly efficient; it only requires the reconstruction of $2 t$ sharings, each towards a single player.

In other words, we can non-robustly but detectably generate $\Omega(n)$ uniform random sharings, unknown to the adversary, with perfect security and communicating $\mathcal{O}\left(n^{2}\right)$ field elements. By now, similarly efficient protocols to generate random sharings are known only with probabilistic checks, which provides a lower level of security and is less elegant.

The novel technique is based on so-called hyper-invertible matrices, i.e., matrices whose every square sub-matrix is invertible. Applying $n$ sharings to such a matrix results in $n$ sharings with the property that (i) if any (up to $t$ ) of the 
inputs sharings are broken, then this can be seen in every subset of $t$ output sharings, and (ii) if any $n-t$ input sharings are uniform random, then every subset of size $n-t$ of output sharings is uniform random.

Using hyper-invertible matrices and some techniques from Bea91a, HMP00, DN07, we construct a perfectly secure multi-party protocol with optimal resilience and linear communication complexity. This can be seen as an efficiency improvement (the most efficient known MPC protocol with perfect security communicates $\mathcal{O}\left(n^{3}\right)$ field elements per multiplication [HMP0] ), or alternatively as a security improvement (the most secure known MPC protocol with linear communication provides error probability [DN07]). In either case, we consider the new protocol to be more elegant, as it employs neither two-dimensional sharings (like all previous perfectly-secure MPC protocols) nor probabilistic checks (like all previous MPC protocols with linear communication complexity).

\section{Preliminaries}

\subsection{Model}

We consider a set $\mathcal{U}$ of users, who can give input and receive output, and a set $\mathcal{P}$ of $n$ players, $\mathcal{P}=\left\{P_{1}, \ldots, P_{n}\right\}$, who perform the computation. The players and users are connected by a complete network of secure (private and authentic) synchronous channels.

The function to be computed is specified as an arithmetic circuit over a finite field $\mathcal{F}$ (with $|\mathcal{F}|>2 n$ ), with input, addition, multiplication, random, and output gates. We denote the number of gates of each type by $c_{I}, c_{A}, c_{M}, c_{R}$, and $c_{O}$, respectively.

The faultiness of players or users is modeled in terms of a central adversary corrupting players and users. The adversary can corrupt up to $t$ players for any fixed $t$ with $t<n / 3$ and any number of users, and make them deviate from the protocol in any desired manner. The adversary is computationally unbounded, active, adaptive, and rushing. The security of our protocols is perfect, i.e., information-theoretic without any error probability.

To every player $P_{i} \in \mathcal{P}$ a unique, non-zero element $\alpha_{i} \in \mathcal{F} \backslash\{0\}$ is assigned.

For the ease of presentation, we always assume that the messages sent through the channels are from the right domain - if a player receives a message which is not in the right domain (e.g., no message at all), he replaces it with an arbitrary message from the specified domain.

\subsection{Byzantine Agreement}

In our multi-party protocol we use Byzantine agreement in both its shapes, broadcast and consensus. Broadcast allows a sender to distribute a value $x$, such that all players receive the same value $x^{\prime}$ (even if the sender is faulty), and $x=x^{\prime}$ if the sender is honest. Consensus allows the players, each holding an 
input $x_{i}$, to reach agreement on a value $x^{\prime}$, where $x=x^{\prime}$ if every honest players holds $x_{i}=x$. For $t<n / 3$, both broadcast and consensus can be simulated with perfect security by a sub-protocol communicating $\mathcal{O}\left(n^{2}\right)$ bits BGP92, CW92. We denote the communication complexity needed for agreeing on a $k$ bit message as $\mathcal{B} \mathcal{A}(k)=n^{2} k$.

\subsection{Player-Elimination Framework}

Player Elimination HMP00 is a general technique, used for constructing efficient MPC protocols. It allows to transform (typically very efficient) non-robust protocols into robust protocols at essentially no additional costs.

The basic idea is to divide the computation into segments and repeat the nonrobust evaluation of each segment until it succeeds, whereby limiting the total number of times the adversary can cause a segment to fail. Each evaluation of a segment proceeds in three steps: (1.) detectable computation (2.) fault detection and (3.) fault localization.

Definition 1. A detectable protocol is a passively secure protocol that can (in the presence of an active adversary) produce incorrect output, however this will be detected by at least one honest player. We say that after detecting a fault the player gets unhappy (sets his happy-bit to unhappy).

In the detectable computation, the actual non-robust (but detectable) protocol is invoked to compute the segment. In the fault detection the players agree on whether or not there are some unhappy players. If all players are happy the computation of the segment was successful, the players keep the output and proceed to the next segment. Otherwise the segment failed, the output is discarded and a pair of players $E=\left\{P_{i}, P_{j}\right\}$ containing at least one corrupted player is localized in the fault localization, eliminated from the actual player set and the segment is repeated with the new player set 1 We denote the original player set as $\mathcal{P}$ (containing $n$ players, up to $t$ of them faulty), and the actual (reduced) player set as $\mathcal{P}^{\prime}$ (containing $n^{\prime}$ players, up to $t^{\prime}$ of them faulty).

By selecting the size of a segment such that there are $t$ segments, the overall costs of the resulting robust protocol are at most twice the costs of the nonrobust protocol (plus the overhead costs for the fault detection and the player elimination).

Special care needs to be taken such that the computation after a (sequence of) player elimination is "compatible" with the outputs of previous segments. We ensure this compatibility be fixing the degree of all sharings to $t$, independent of the actual threshold $t^{\prime}$. Note that a sharing (among $\mathcal{P}^{\prime}$ ) of degree $t$ can be reconstructed as long as $t+2 t^{\prime}<n^{\prime}$, what is clearly satisfied when $t<n / 3$.

Technically, a player-elimination protocol proceeds as follows:

\footnotetext{
${ }^{1}$ Note that we eliminate players and not users. If a party playing the role of a player as well as the role of a user is eliminated from the player set, it still keeps its user role - can give input and receive output.
} 


\section{Protocol with Player-Elimination}

Let $\mathcal{P}^{\prime} \leftarrow \mathcal{P}, n^{\prime} \leftarrow n, t^{\prime} \leftarrow t$. Divide computation into $t$ segments of similar size, and do the following for each segment:

0. Every $P_{i} \in \mathcal{P}^{\prime}$ sets his happy-bit to happy (i.e., $P_{i}$ did not observe a fault).

1. Detectable Computation: Compute the actual segment in detectable manner, such that (i) if all players in $\mathcal{P}^{\prime}$ follow their protocol, then the computation succeeds and all players remain happy, and (ii) if the output is incorrect, then at least one honest player in $\mathcal{P}^{\prime}$ detects so and gets unhappy.

2. Fault Detection: Reach agreement on whether or not all players in $\mathcal{P}^{\prime}$ are happy (involves Byzantine Agreement). If all players are happy, proceed with the next segment. If at least one player is unhappy, proceed with the following fault-localization procedure.

3. Fault Localization: Find $E \subseteq \mathcal{P}^{\prime}$ with $|E|=2$, containing at least one corrupted player.

4. Player Elimination: Set $\mathcal{P}^{\prime} \leftarrow \mathcal{P}^{\prime} \backslash E, n^{\prime} \leftarrow n^{\prime}-2, t^{\prime} \leftarrow t^{\prime}-1$, and repeat the segment.

\subsection{Circuit Randomization}

Circuit randomization Bea91a allows to compute a sharing $[z]$ of the product $z$ of two factors $x$ and $y$, shared as $[x]$ and $[y]$, at the costs of two public reconstructions, when a pre-shared random triple $([a],[b],[c])$ with $c=a b$ is available. This technique allows to first prepare $c_{M}$ shared multiplication triples $([a],[b],[c])$, and then to evaluate a circuit with $c_{M}$ multiplication by a sequence of public reconstructions.

The trick of circuit randomization is that $z=x y$ can be expressed as $z=$ $((x-a)+a)((y-b)+b)$, hence $z=d e+d b+a e+c$, where $(a, b, c)$ is a multiplication triple and $d=x-a$ and $e=y-b$. For a random multiplication triple, $d$ and $e$ are random values independent of $x$ and $y$, hence a sharing $[z]$ can be linearly computed as $[z]=[d e]+d[b]+e[a]+[c]$, by reconstructing $[d]=[x]-[a]$ and $[e]=[y]-[b]$.

\section{Hyper-invertible Matrices}

\subsection{Definition}

A hyper-invertible matrix is a matrix of which every (non-trivial) square submatrix is invertible.

Definition 2. An r-by-c matrix $M$ is hyper-invertible if for any index sets $R \subseteq$ $\{1, \ldots, r\}$ and $C \subseteq\{1, \ldots, c\}$ with $|R|=|C|>0$, the matrix $M_{R}^{C}$ is invertible, where $M_{R}$ denotes the matrix consisting of the rows $i \in R$ of $M, M^{C}$ denotes the matrix consisting of the columns $j \in C$ of $M$, and $M_{R}^{C}=\left(M_{R}\right)^{C}$. 


\subsection{Construction}

We present a construction of a hyper-invertible $n$-by- $n$ matrix $M$ over a finite field $\mathcal{F}$ with $|\mathcal{F}| \geq 2 n$. A hyper-invertible $r$-by- $c$ matrix can be extracted as a sub-matrix of such a matrix with $n=\max (r, c)$.

Construction 1. Let $\alpha_{1}, \ldots, \alpha_{n}, \beta_{1}, \ldots, \beta_{n}$ denote fixed distinct elements in $\mathcal{F}$, and consider the function $f: \mathcal{F}^{n} \rightarrow \mathcal{F}^{n}$, mapping $\left(x_{1}, \ldots, x_{n}\right)$ to $\left(y_{1}, \ldots, y_{n}\right)$ such that the points $\left(\beta_{1}, y_{1}\right), \ldots,\left(\beta_{n}, y_{n}\right)$ lie on the polynomial $g(\cdot)$ of degree $n-1$ defined by the points $\left(\alpha_{1}, x_{1}\right), \ldots,\left(\alpha_{n}, x_{n}\right)$. Due to the linearity of Lagrange interpolation, $f$ is linear and can be expressed as a matrix $M=\left\{\lambda_{i, j}\right\}_{i=1, \ldots, n}^{j=1, \ldots n}$, where $\lambda_{i, j}=\prod_{\substack{k=1 \\ k \neq j}}^{n} \frac{\beta_{i}-\alpha_{k}}{\alpha_{j}-\alpha_{k}}$.

Lemma 1. Construction 1 yields a hyper-invertible n-by-n matrix $M$.

Proof. We have to show that for any index sets $R, C \subseteq\{1, \ldots, n\}$ with $|R|=$ $|C|>0, M_{R}^{C}$ is invertible. As $|R|=|C|$, it is sufficient to show that the mapping defined by $M_{R}^{C}$ is surjective, i.e., for every $\vec{y}_{R}$ there exists an $\vec{x}_{C}$ such that $\vec{y}_{R}=$ $M_{R}^{C} \vec{x}_{C}$. Equivalently, we show that for every $\vec{y}_{R}$ there exists an $\vec{x}$ such that $\vec{y}_{R}=$ $M_{R} \vec{x}$ and $\vec{x}_{\bar{C}}=\overrightarrow{0}$, where $\bar{C}=\{1, \ldots, n\} \backslash C$. Remember that $M$ is defined such that the points $\left(\alpha_{1}, x_{1}\right), \ldots,\left(\alpha_{n}, x_{n}\right),\left(\beta_{1}, y_{1}\right), \ldots,\left(\beta_{n}, y_{n}\right)$ lie on a polynomial $g(\cdot)$ of degree $n-1$. Given the $n$ points $\left\{\left(\alpha_{j}, 0\right)\right\}_{j \notin C}$ and $\left\{\left(\beta_{i}, y_{i}\right)\right\}_{i \in R}$, the polynomial $g(\cdot)$ can be determined by Lagrange interpolation, and $\vec{x}_{C}$ can be computed linearly from $\vec{y}_{R}$. Hence, $M_{R}^{C}$ is invertible.

\subsection{Properties}

The mappings defined by hyper-invertible matrices have a very nice symmetry property: Any subset of $n$ input/output values can be expressed as a linear function of the remaining $n$ input/output values:

Lemma 2. Let $M$ be a hyper-invertible $n$-by-n matrix and $\left(y_{1}, \ldots, y_{n}\right)=$ $M\left(x_{1}, \ldots, x_{n}\right)$. Then for any index sets $A, B \subseteq\{1, \ldots, n\}$ with $|A|+|B|=n$, there exists an invertible linear function $f: \mathcal{F}^{n} \rightarrow \mathcal{F}^{n}$, mapping the values $\left\{x_{i}\right\}_{i \in A},\left\{y_{i}\right\}_{i \in B}$ onto the values $\left\{x_{i}\right\}_{i \notin A},\left\{y_{i}\right\}_{i \notin B}$.

Proof. We have $\vec{y}=M \vec{x}$ and $\vec{y}_{B}=M_{B} \vec{x}=M_{B}^{A} \vec{x}_{A}+M_{B}^{\bar{A}} \vec{x}_{\bar{A}}$. Due to hyperinvertibility, $M_{B}^{\bar{A}}$ is invertible, and $\vec{x}_{\bar{A}}=\left(M_{B}^{\bar{A}}\right)^{-1}\left(\vec{y}_{B}-M_{B}^{A} \vec{x}_{A}\right) \cdot \vec{y}_{\bar{B}}$ can be computed similarly.

\section{Protocol Overview}

The new MPC protocol proceeds in two phases: the preparation phase and the computation phase.

In the preparation phase, degree- $t$ sharings of random $(a, b, c)$-triples are generated (in parallel), one for every multiplication gate. Furthermore, for every 
random gate as well as for every input gate, a $t$-sharing of a random $r$ is generated. For the sake of simplicity, we generate $c_{M}+c_{R}+c_{I}$ random triples, where for random and input gates, only the first component is used. The preparation phase makes use of the player-elimination technique.

In the computation phase, the actual circuit is computed. Input gates are evaluated with help of a pre-shared random value $r$. Due to the linearity of the used secret-sharing, the linear gates can be computed locally - without communication. Random gates are evaluated simply by picking an unused pre-shared random value $r$. Multiplication gates are evaluated with help of one prepared $(a, b, c)$-triple, using Beaver's circuit randomization technique Bea91a. Output gates involve a (robust) secret reconstruction.

\section{$5 \quad$ Secret Sharing}

\subsection{Definitions and Notation}

As secret-sharing scheme, we use the standard Shamir sharing scheme [Sha79].

Definition 3. We say that a value $s$ is (correctly) $d$-shared (among the players in $\left.\mathcal{P}^{\prime}\right)$ if every honest player $P_{i} \in \mathcal{P}^{\prime}$ is holding a share $s_{i}$ of $s$, such that there exists a degree-d polynomial $p(\cdot)$ with $p(0)=s$ and $p\left(\alpha_{i}\right)=s_{i}$ for every $P_{i} \in \mathcal{P}^{\prime}$ The vector $\left(s_{1}, \ldots, s_{n^{\prime}}\right)$ of shares is called a d-sharing of $s$, and is denoted by $[s]_{d}$. A (possibly incomplete) set of shares is called $d$-consistent if these shares lie on a degree $d$ polynomial.

Most of the sharings used in our protocol are $t$-sharings - denoted as $[\cdot]_{t}$. In the preparation phase we also temporarily use $t^{\prime}$ - and $2 t^{\prime}$-sharings (denoted by $[\cdot]_{t^{\prime}}$ and $[\cdot]_{2 t^{\prime}}$, respectively).

By saying that the players in $\mathcal{P}^{\prime}$ compute (locally) $\left(\left[y^{(1)}\right]_{d^{\prime}}, \ldots,\left[y^{\left(m^{\prime}\right)}\right]_{d^{\prime}}\right)=$ $f\left(\left[x^{(1)}\right]_{d}, \ldots,\left[x^{(m)}\right]_{d}\right.$ ) (for any function $f: \mathcal{F}^{m} \rightarrow \mathcal{F}^{m^{\prime}}$ ) we mean that every player $P_{i}$ applies this function to his shares, i.e. computes $\left(y_{i}^{(1)}, \ldots, y_{i}^{\left(m^{\prime}\right)}\right)=$ $f\left(x_{i}^{(1)}, \ldots, x_{i}^{(m)}\right)$. Note that by applying any linear function to correct $d$-sharings we get a correct $d$-sharing of the output. However, by multiplying two correct $d$-sharings we get a correct $2 d$-sharing of the product, i.e. $[a]_{d}[b]_{d}=[a b]_{2 d}$.

\subsection{The Share Protocol}

The following (trivial) Share protocol allows an honest dealer $P_{D}$ to correctly $d$-share a secret $s$ among the players in $\mathcal{P}^{\prime}$, while communicating $\mathcal{O}(n \kappa)$ bits. We stress that this protocol does not ensure that the resulting sharing is consistent; a corrupted dealer might distribute totally inconsistent shares. The consistency of sharings must be verified separately.

Protocol Share $\left(P_{D} \in(\mathcal{P} \cup \mathcal{U}), s, d\right)$

1. $P_{D}$ chooses a random degree- $d$ polynomial $p(\cdot)$ with $s=p(0)$ and sends $s_{i}=p\left(\alpha_{i}\right)$ to every $P_{i} \in \mathcal{P}^{\prime}$.

\footnotetext{
${ }^{2}$ Where $\alpha_{i}$ denotes the unique fixed value assigned to $P_{i}$.
} 


\subsection{The Reconstruct Protocols}

We use two reconstruction protocols: one for private and one for public reconstruction. Both can be either robust or only detectable - depending on the degree of the sharings to be reconstructed.

In the private reconstruction protocol the players simply send their shares to the receiver $P_{R}$ (a player or a user) who interpolates the secret (if possible).

\section{Protocol ReconsPriv $\left(P_{R} \in(\mathcal{P} \cup \mathcal{U}), d,[s]_{d}\right)$}

1. Every player $P_{i} \in \mathcal{P}^{\prime}$ sends his share $s_{i}$ of $s$ to $P_{R}$.

2. If there exists a degree- $d$ polynomial $p(\cdot)$ such that at least $d+t^{\prime}+1$ of the received shares lie on it, then $P_{R}$ computes the secret $s=p(0)$. Otherwise $P_{R}$ gets unhappy.

Lemma 3. For $d<n^{\prime}-2 t^{\prime}$, the protocol ReconsPriv robustly reconstructs $[s]_{d}$ towards $P_{R}$. For $d<n^{\prime}-t^{\prime}$, ReconsPriv detectably reconstructs $[s]_{d}$ towards $P_{R}$ (i.e., $P_{R}$ either outputs $s$ or gets unhappy, where the latter only happens when some players are faulty). ReconsPriv communicates $\mathcal{O}(n \kappa)$ bits.

The public reconstruction protocol ReconsPubl takes $T=n^{\prime}-2 t^{\prime}=n-2 t=\Omega(n)$ correct $d$-sharings $\left[s_{1}\right]_{d}, \ldots,\left[s_{T}\right]_{d}$ and publicly (to all players in $\mathcal{P}^{\prime}$ ) outputs the (correct) values $s_{1}, \ldots, s_{T}$ or fails (with at least one honest player being unhappy). In ReconsPubl we use the idea of DN07: first the $T$ sharings $\left[s_{1}\right]_{d}, \ldots,\left[s_{T}\right]_{d}$ are expanded (using a linear error-correcting code) to $n^{\prime}$ sharings $\left[u_{1}\right]_{d}, \ldots,\left[u_{n^{\prime}}\right]_{d} 3^{3}$ each of which is reconstructed towards one player in $\mathcal{P}^{\prime}$ (using ReconsPriv). Then every $P_{i} \in \mathcal{P}^{\prime}$ sends his reconstructed value $u_{i}$ to every other player in $\mathcal{P}^{\prime}$, who tries to decode (with error correction) the received code word $\left(u_{1}, \ldots, u_{n^{\prime}}\right)$ to $s_{1}, \ldots, s_{T}$. ReconsPubl communicates $\mathcal{O}\left(n^{2} \kappa\right)$ bits to reconstruct $T=\Omega(n)$ sharings.

Protocol ReconsPubl $\left(d,\left[s_{1}\right]_{d}, \ldots,\left[s_{T}\right]_{d}\right)$

1. For every $j=1, \ldots, n^{\prime}$ the players in $\mathcal{P}^{\prime}$ (locally) compute $\left[u_{j}\right]_{d}$ as:

$$
\left[u_{j}\right]_{d}=\left[s_{1}\right]_{d}+\left[s_{2}\right]_{d} \beta_{j}+\left[s_{3}\right]_{d} \beta_{j}^{2}+\ldots+\left[s_{T}\right]_{d} \beta_{j}^{T-1}
$$

2. For every $P_{i} \in \mathcal{P}^{\prime}$, ReconsPriv is invoked to reconstruct $\left[u_{i}\right]_{d}$ towards $P_{i}$.

3. Every $P_{i} \in \mathcal{P}^{\prime}$ sends $u_{i}$ (or $\perp$ if unhappy) to every $P_{j} \in \mathcal{P}^{\prime}$.

4. $\forall P_{i} \in \mathcal{P}^{\prime}$ : If $P_{i}$ received at least $T+t^{\prime} \quad(T-1)$-consistent values (in the previous step), he computes $s_{1}, \ldots, s_{T}$ from any $T$ of them. Otherwise he gets unhappy.

Lemma 4. For $d<n^{\prime}-2 t^{\prime}$, the protocol ReconsPubl robustly reconstructs $\left[s_{1}\right]_{d}, \ldots,\left[s_{T}\right]_{d}$ towards all players in $\mathcal{P}^{\prime}$. For $d<n^{\prime}-t^{\prime}$, ReconsPubl detectably reconstructs $\left[s_{1}\right]_{d}, \ldots,\left[s_{T}\right]_{d}$ towards all players in $\mathcal{P}^{\prime}$ (i.e., every $P_{i} \in \mathcal{P}^{\prime}$ either outputs $s_{1}, \ldots, s_{T}$ or gets unhappy, where the latter only happens when some players are faulty). ReconsPubl communicates $\mathcal{O}\left(n^{2} \kappa\right)$ bits.

${ }^{3}$ For this we interpret $s_{1}, \ldots, s_{T}$ as coefficients of a degree $T-1$ polynomial and $u_{1}, \ldots, u_{n^{\prime}}$ as evaluations of this polynomial at $n^{\prime}$ fixed positions $\beta_{1}, \ldots, \beta_{n^{\prime}}$. 


\section{Preparation Phase}

\subsection{Overview}

The goal of the preparation phase is to generate correct $t$-sharings of $c_{M}+c_{R}+c_{I}$ secret random triples $\left(a_{k}, b_{k}, c_{k}\right)$, such that $c_{k}=a_{k} b_{k}$ for $k=1, \ldots, c_{M}+c_{R}+c_{I}$. We stress that all resulting sharings must be $t$-sharings (rather than $t^{\prime}$-sharings) among the player set $\mathcal{P}^{\prime} 4$

The preparation phase uses player elimination, i.e. the generation of the triples is divided into $t$ segments of length $\ell=\left\lceil\frac{c_{M}+c_{R}+c_{I}}{t}\right\rceil$. In every segment the nonrobust protocol GenerateTriples is invoked, which either generates correct triples, or fails with at least one honest player being unhappy.

The generation of the triples follows the approach of [DN07]: First, the players generate random $a$ and $b$ values, both simultaneously shared with degree $t$ (for outputting) and degree $t^{\prime}$ (for multiplication). Additionally, the players generate random value $r$, simultaneously shared with degree $t$ and degree $2 t^{\prime}$. Then, they locally compute the $2 t^{\prime}$-sharing $[a b]_{2 t^{\prime}}$ (by every player multiplying his respective shares), publicly reconstruct the difference $[a b]_{2 t^{\prime}}-[r]_{2 t^{\prime}}$ and add it (locally) to $[r]_{t}$, resulting in $[a b]_{t}$. Finally, the players output the triple $\left([a]_{t},[b]_{t},[a b]_{t}\right)$.

Definition 4. A value $x$ is $\left(d, d^{\prime}\right)$-shared among the players $\mathcal{P}^{\prime}$, denoted as $[x]_{d, d^{\prime}}$, if $x$ is both $d$-shared and $d^{\prime}$-shared. We denote such a sharing as a doublesharing, and the pair of shares held by each player as his double-share.

We (trivially) observe that the sum of correct $\left(d, d^{\prime}\right)$-sharings is a correct $\left(d, d^{\prime}\right)$ sharing of the sum.

\subsection{Generating Random Double-Sharings}

The following non-robust protocol DoubleShareRandom $\left(d, d^{\prime}\right)$ either generates $T$ independent secret random values $r_{1}, \ldots, r_{T}$, each independently $\left(d, d^{\prime}\right)$-shared among $\mathcal{P}^{\prime}$, or fails with at least one honest player being unhappy.

The generation of the random double-sharings employs hyper-invertible matrices: First, every player $P_{i} \in \mathcal{P}^{\prime}$ selects and double-shares a random value $s_{i}$. Then, the players compute double-sharings of the values $r_{i}$, defined as $\left(r_{1}, \ldots, r_{n^{\prime}}\right)=M\left(s_{1}, \ldots, s_{n^{\prime}}\right)$, where $M$ is a hyper-invertible $n^{\prime}$-by- $n^{\prime}$ matrix. $2 t^{\prime}$ of the resulting double-sharings are reconstructed, each towards a different player, who verify the correctness of the double-sharings (and gets unhappy in case of a fault). The remaining $n^{\prime}-2 t^{\prime}=T$ double-sharings are outputted. This procedure guarantees that if all honest players are happy, then at least $n^{\prime}$ double-sharings are correct (the $n^{\prime}-t^{\prime}$ double-sharings inputted by honest players, as well as the $t^{\prime}$ double-sharings verified by honest players), and due to the hyper-invertibility of $M$, all $2 n^{\prime}$ double-sharings must be correct (the remaining double-sharings can be computed linearly from the good double-sharings).

${ }^{4}$ Remember that as $t \leq n^{\prime}-2 t^{\prime}$ (according to Lemma 3 and 44), such sharings can be robustly reconstructed (regardless of the actual player set $\mathcal{P}^{\prime}$ ). 
Furthermore, the outputted double-sharings are random and unknown to the adversary, as there is a bijective mapping from any $T$ double-sharings inputted by honest players to the outputted double-sharings.

\section{Protocol DoubleShareRandom $\left(d, d^{\prime}\right)$}

1. Secret Share: Every $P_{i} \in \mathcal{P}^{\prime}$ chooses a random $s_{i}$ and acts (twice in parallel) as a dealer in Share to distribute the shares among the players in $\mathcal{P}^{\prime}$, resulting in $\left[s_{i}\right]_{d, d^{\prime}}$.

2. Apply $M$ : The players in $\mathcal{P}^{\prime}$ (locally) compute $\left(\left[r_{1}\right]_{d, d^{\prime}}, \ldots,\left[r_{n^{\prime}}\right]_{d, d^{\prime}}\right)=$ $M\left(\left[s_{1}\right]_{d, d^{\prime}}, \ldots,\left[s_{n^{\prime}}\right]_{d, d^{\prime}}\right)$. In order to do so, every $P_{i}$ computes his doubleshare of each $r_{j}$ as linear combination of his double-shares of the $s_{k}$-values.

3. CHECK: For $i=T+1, \ldots, n^{\prime}$, every $P_{j} \in \mathcal{P}^{\prime}$ sends his double-share of $\left[s_{i}\right]_{d, d^{\prime}}$ to $P_{i}$, who checks that all $n^{\prime}$ double-shares define a correct double-sharing of some value $s_{i}$. More precisely, $P_{i}$ checks that all $d$-shares indeed lie on a polynomial $g(\cdot)$ of degree $d$, and that all $d^{\prime}$-shares indeed lie on a polynomial $g^{\prime}(\cdot)$ of degree $d^{\prime}$, and that $g(0)=g^{\prime}(0)$. If any of the checks fails, $P_{i}$ gets unhappy.

4. Output: The remaining $T$ double-sharings $\left[r_{1}\right]_{d, d^{\prime}}, \ldots,\left[r_{T}\right]_{d, d^{\prime}}$ are outputted.

Lemma 5. If DoubleShareRandom $\left(d, d^{\prime}\right)$ succeeds (i.e., all honest players are happy), it outputs $T=n^{\prime}-2 t^{\prime}$ correct and random $\left(d, d^{\prime}\right)$-sharings (among $\left.\mathcal{P}^{\prime}\right)$, unknown to the adversary. DoubleShareRandom communicates $\mathcal{O}\left(n^{2} \kappa\right)$ bits to generate $\Omega(n)$ double-sharings.

Proof. Correctness: Assume that all honest players remain happy during the protocol. Then for all honest $P_{i}$ with $i \in\left\{T+1, \ldots, n^{\prime}\right\}$, the sharing of $r_{i}$ checked by $P_{i}$ in Step 3 is a correct $\left(d, d^{\prime}\right)$-sharing. As $T=n^{\prime}-2 t^{\prime}$, there are at least $t^{\prime}$ correct sharings of the values $r_{k}$. Furthermore, every sharing of an $s_{i}$ distributed by an honest $P_{i}$ in Step 1 is a correct $\left(d, d^{\prime}\right)$-sharing. Thus there are at least $n^{\prime}-t^{\prime}$ correct sharings of the values $s_{k}$. Given these (at least) $n^{\prime}$ correct $\left(d, d^{\prime}\right)$ sharings, the sharings of all other values $s_{k}$ and $r_{k}$ can be computed linearly. As a linear combination of a correct $\left(d, d^{\prime}\right)$-sharing is again a $\left(d, d^{\prime}\right)$-sharing, it follows that all values $s_{1}, \ldots, s_{n^{\prime}}, r_{1}, \ldots, r_{n^{\prime}}$ are correctly $\left(d, d^{\prime}\right)$-shared.

PRIVACY: The adversary knows (at most) $t^{\prime}$ of the input sharings $s_{k}$ (those provided by corrupted players), and $t^{\prime}$ of the output sharings $r_{k}$ (with $k>T$, those reconstructed towards corrupted players). When fixing these $2 t^{\prime}$ sharings, then there exists a bijective mapping between any other (honest) $T$ input sharings and the first $T$ output sharings (Lemma 21), hence the sharings $\left[r_{1}\right]_{d, d^{\prime}}, \ldots,\left[r_{T}\right]_{d, d^{\prime}}$ are uniformly at random, unknown to the adversary.

Communication: The stated communication can easily be verified by inspecting the protocol.

\subsection{Generating Random Triples}

Now we present the non-robust protocol GenerateTriples that either generates $T=n^{\prime}-2 t^{\prime}$ correctly $t$-shared $(a, b, c)$-triples, or fails (with at least one honest 
player being unhappy). The idea of the protocol GenerateTriples is the following: First DoubleShareRandom is invoked 3 times to generated the random doublesharings $\left[a_{1}\right]_{t, t^{\prime}}, \ldots,\left[a_{T}\right]_{t, t^{\prime}},\left[b_{1}\right]_{t, t^{\prime}}, \ldots,\left[b_{T}\right]_{t, t^{\prime}}$, and $\left[r_{1}\right]_{t, 2 t^{\prime}}, \ldots,\left[r_{T}\right]_{t, 2 t^{\prime}}$, respectively. Then for every pair $a_{k}, b_{k}$, a $t$-sharing of the product $c_{k}=a_{k} b_{k}$ is computed by reducing the locally computed $2 t^{\prime}$-sharing $\left[c_{k}\right]_{2 t^{\prime}}=\left[a_{k}\right]_{t^{\prime}}\left[b_{k}\right]_{t^{\prime}}$ to a $t$-sharing $\left[c_{k}\right]_{t}$ using the $t$-sharing $\left[r_{k}\right]_{t}$ and the $2 t^{\prime}$-sharing $\left[r_{k}\right]_{2 t^{\prime}}$ of the random value $r_{k}$.

\section{Protocol GenerateTriples}

1. Generate Double-Sharings: Invoke DoubleShareRandom three times in parallel to generate the double-sharings $\left[a_{1}\right]_{t, t^{\prime}}, \ldots,\left[a_{T}\right]_{t, t^{\prime}}$, $\left[b_{1}\right]_{t, t^{\prime}}, \ldots,\left[b_{T}\right]_{t, t^{\prime}}$, and $\left[r_{1}\right]_{t, 2 t^{\prime}}, \ldots,\left[r_{T}\right]_{t, 2 t^{\prime}}$.

2. Multiply:

2.1 For $k=1, \ldots, T$, the players in $\mathcal{P}^{\prime}$ compute (locally) the $2 t^{\prime}$-sharing $\left[c_{k}\right]_{2 t^{\prime}}$ of $c_{k}=a_{k} b_{k}$ as $\left[c_{k}\right]_{2 t^{\prime}}=\left[a_{k}\right]_{t^{\prime}}\left[b_{k}\right]_{t^{\prime}}$ (by every player computing the product of his shares).

2.2 For $k=1, \ldots, T$, the players in $\mathcal{P}^{\prime}$ compute (locally) a $2 t^{\prime}$-sharing of the difference $\left[d_{k}\right]_{2 t^{\prime}}=\left[c_{k}\right]_{2 t^{\prime}}-\left[r_{k}\right]_{2 t^{\prime}}$

2.3 Invoke ReconsPubl $\left(\mathcal{R}=\mathcal{P}^{\prime}, d=2 t^{\prime},\left[d_{1}\right]_{2 t^{\prime}}, \ldots,\left[d_{T}\right]_{2 t^{\prime}}\right)$ to reconstruct $d_{1}, \ldots, d_{T}$ towards every player in $\mathcal{P}^{\prime}$.

2.4 For $k=1, \ldots, T$, the players in $\mathcal{P}^{\prime}$ compute (locally) the $t$-sharing $\left[c_{k}\right]_{t}=\left[r_{k}\right]_{t}+\left[d_{k}\right]_{0}$, where $\left[d_{k}\right]_{0}$ denotes the constant sharing $\left[d_{k}\right]_{0}=$ $\left(d_{k}, \ldots, d_{k}\right)$.

3. Output: The $t$-shared triples $\left(\left[a_{1}\right]_{t},\left[b_{1}\right]_{t},\left[c_{1}\right]_{t}\right), \ldots,\left(\left[a_{T}\right]_{t},\left[b_{T}\right]_{t},\left[c_{T}\right]_{t}\right)$ are outputted.

Lemma 6. If GenerateTriples succeeds (i.e., all honest players are happy), it outputs independent random t-sharings of $T=\Omega(n)$ random triples $\left(a_{1}, b_{1}, c_{1}\right), \ldots,\left(a_{T}, b_{T}, c_{T}\right)$ with $a_{k}, b_{k}$ independent uniform random values and $c_{k}=a_{k} b_{k}$ for $k=1, \ldots, T$. GenerateTriples communicates $\mathcal{O}\left(n^{2} \kappa\right)$ bits.

Proof. The security of GenerateTriples follows directly from the security of DoubleShareRandom.

\subsection{Preparation Phase - Main Protocol}

The following protocol PreparationPhase divides the generation of the $c_{M}+c_{R}+c_{I}$ triples into $t$ segments of length $\ell=\left\lceil\frac{c_{M}+c_{R}+c_{I}}{t}\right\rceil$. In each segment the triples are generated invoking the non-robust protocol GenerateTriples (as often as necessary), then the players reach agreement on whether or not all players are happy. If yes, they proceed to the next segment. Otherwise, a pair of players is identified in FaultLocalization, excluded from the actual player set $\mathcal{P}^{\prime}$ and the segment is repeated (with the new $\mathcal{P}^{\prime}$ and all players setting their happy-bit to happy). 


\section{Protocol PreparationPhase}

For each segment $k=1, \ldots, t$ do:

0 . Every $P_{i} \in \mathcal{P}^{\prime}$ sets his happy-bit to happy.

1. Triple Generation: Invoke GenerateTriples $\left\lceil\frac{\ell}{T}\right\rceil$ times in parallel.

2. Fault Detection: Reach agreement whether or not at least one player is unhappy:

2.1 Every $P_{i} \in \mathcal{P}^{\prime}$ sends his happy-bit to every $P_{j} \in \mathcal{P}^{\prime}$, who gets unhappy if at least one $P_{i}$ claims to be unhappy.

2.2 The players in $\mathcal{P}^{\prime}$ run a consensus protocol on their respective happybits. If the consensus outputs "happy", then the generated triples are outputted and the segment is finished. Otherwise, the following FaultLocalization step is executed.

3. Fault Localization: Localize $E \subseteq \mathcal{P}^{\prime}$ with $|E|=2$ and at least one player in $E$ being corrupted:

3.0 Denote the player $P_{r} \in \mathcal{P}^{\prime}$ with the smallest index $r$ as the referee 5

3.1 Every $P_{i} \in \mathcal{P}^{\prime}$ sends everything he received and all random values he chose during the computation of the actual segment (including fault detection) to $P_{r}$.

3.2 Given the values received in Step 3.1, $P_{r}$ can reproduce every message that should have been sent (by applying the respective protocol instructions of the sender), and compare it with the value that the recipient claims to have received. Then $P_{r}$ broadcasts $\left(l, i, j, x, x^{\prime}\right)$, where $l$ is the index of a message where $P_{i}$ should have sent $x$ to $P_{j}$, but $P_{j}$ claims to have received $x^{\prime} \neq x$.

3.3 The accused players broadcast whether they agree with $P_{r}$. If $P_{i}$ disagrees, set $E=\left\{P_{r}, P_{i}\right\}$, if $P_{j}$ disagrees, set $E=\left\{P_{r}, P_{j}\right\}$, otherwise set $E=\left\{P_{i}, P_{j}\right\}$.

4. Player Elimination: Set $\mathcal{P}^{\prime} \leftarrow \mathcal{P}^{\prime} \backslash E, n^{\prime} \leftarrow n^{\prime}-2, t^{\prime} \leftarrow t^{\prime}-1$, and repeat the segment.

Lemma 7. The protocol PreparationPhase generates independent random $t$ sharings of $c_{M}+c_{R}+c_{I}$ secret triples $\left(a_{k}, b_{k}, c_{k}\right)$ with $a_{k}, b_{k}$ independent uniform random values and $c_{k}=a_{k} b_{k}$ for $k=1, \ldots, c_{M}+c_{R}+c_{I}$. PreparationPhase communicates $\mathcal{O}\left(\left(c_{M}+c_{R}+c_{I}\right) n \kappa+n^{2} \kappa+t \mathcal{B} \mathcal{A}(\kappa)\right)$ bits, which amounts to $\mathcal{O}\left(\left(c_{M}+c_{R}+c_{I}\right) n \kappa+n^{3} \kappa\right)$ bits overall.

\section{Computation Phase}

In the computation phase, the circuit is robustly evaluated, whereby all intermediate values are $t$-shared among the players in $\mathcal{P}^{\prime}$.

Input gates are realized by reconstructing a pre-shared random value $r$ towards the input-providing user, who then broadcasts the difference of this $r$ and his input.

${ }^{5}$ The communication can be balanced by selecting a player who has not yet been referee in a previous segment. 
Due to the linearity of the secret-sharing scheme, linear gates can be computed locally simply by applying the linear function to the shares, i.e. for any linear function $f(\cdot, \cdot)$, a sharing $[c]=[f(a, b)]$ is computed by letting every player $P_{i}$ compute $c_{i}=f\left(a_{i}, b_{i}\right)$.

With every random gate, one random sharing $[r]$ (from the preparation phase) is associated and $[r]_{t}$ is directly used as outcome of the random gate.

With every multiplication gate, one $([a],[b],[c])$-triple (from the preparation phase) is associated, which is used to compute a sharing of the product at the cost of two public reconstruction. For the sake of efficiency, we evaluate $T / 2$ multiplication gates at once (such that we can publicly reconstruct $T$ sharings at once). This of course requires that these multiplication gates do not depend on each other, i.e., that they all have the same multiplicative depth in the circuit 6

Output gates involve a (robust) secret reconstruction.

\section{Protocol ComputationPhase}

Evaluate the gates of the circuit as follows:

- Input Gate (User $U$ inputs $s$ ):

1. Reconstruct the associated sharing $[r]_{t}$ towards $U$ with $\operatorname{ReconsPriv}(U, t,[r])$. This is robust because $t<n^{\prime}-2 t^{\prime}$.

2. User $U$ computes and broadcasts the difference $d=s-r$.

3. Every $P_{i} \in \mathcal{P}^{\prime}$ computes his share $s_{i}$ of $s$ locally as $s_{i}=d+r_{i}$.

- Addition/Linear Gate: Every $P_{i} \in \mathcal{P}^{\prime}$ applies the linear function on his respective shares.

- Random Gate: Pick the sharing $[r]_{t}$ associated with the gate.

- Multiplication Gate: Up to $\lfloor T / 2\rfloor$ (where $T=n-2 t$ ) multiplication gates are processed simultaneously. Denote the factor sharings as $\left(\left[x_{1}\right],\left[y_{1}\right]\right), \ldots,\left(\left[x_{T / 2}\right],\left[y_{T / 2}\right]\right)$, and the associated triples as $\left(\left[a_{1}\right],\left[b_{1}\right],\left[c_{1}\right]\right), \ldots,\left(\left[a_{T / 2}\right],\left[b_{T / 2}\right],\left[c_{T / 2}\right]\right)$. The products $\left[z_{1}\right], \ldots,\left[z_{T / 2}\right]$ are computed as follows:

1. For $k=1, \ldots, T / 2$, the players compute $\left[d_{k}\right]=\left[x_{k}\right]-\left[a_{k}\right]$ and $\left[e_{k}\right]=$ $\left[y_{k}\right]-\left[b_{k}\right]$.

2. Invoke ReconsPubl to publicly reconstruct the $T$-sharings $\left(d_{1}, e_{1}\right), \ldots,\left(d_{T / 2}, e_{T / 2}\right)$. Note that this is robust, as $t<n^{\prime}-2 t^{\prime}$.

3. For $k=1, \ldots, T / 2$, the players compute the product sharings $\left[z_{k}\right]_{t}=$ $[d e]_{0}+d[b]_{t}+e[a]_{t}+[c]_{t}$, where $[d e]_{0}$ denotes the (implicitly defined) 0 -sharing of de.

- Output Gate (output $[s]$ to User $U$ ): Invoke ReconsPriv $\left(U, t,[s]_{t}\right)$.

Lemma 8. The protocol ComputationPhase perfectly securely evaluates a circuit with $c_{I}$ input, $c_{R}$ random, $c_{M}$ multiplication, and $c_{O}$ output gates, given $c_{I}+c_{R}+c_{M}$ pre-shared random multiplication triples, with communicating

\footnotetext{
${ }^{6}$ The multiplicative depth of a gate is the maximum number of multiplication gates
} on any path from input/random gates to this gate. 
$\mathcal{O}\left(\left(c_{I} n+c_{M} n+c_{O} n+D_{M} n^{2}\right) \kappa+c_{I} \mathcal{B} \mathcal{A}(\kappa)\right)$ bits, where $D_{M}$ denotes the multiplicative depth of the circuit.

Theorem 1. The MPC protocol consisting of PreparationPhase and ComputationPhase evaluates a circuit with $c_{I}$ input, $c_{R}$ random, $c_{M}$ multiplication, and $c_{O}$ output gates, with communicating $\mathcal{O}\left(\left(c_{I} n+c_{R} n+c_{M} n+c_{O} n+\right.\right.$ $\left.\left.D_{M} n^{2}\right) \kappa+\left(c_{I}+n\right) \mathcal{B} \mathcal{A}(\kappa)\right)$ bits, which amounts to $\mathcal{O}\left(\left(c_{I} n^{2}+c_{R} n+c_{M} n+\right.\right.$ $\left.\left.c_{O} n+D_{M} n^{2}\right) \kappa+n^{3} \kappa\right)$ bits, where $D_{M}$ denotes the multiplicative depth of the circuit. The protocol is perfectly secure against an active adversary corrupting $t<n / 3$ players.

The communication complexity for giving input can be improved from $\mathcal{O}\left(n^{2} \kappa\right)$ per input to $\mathcal{O}(n \kappa)$. Details can be found in Appendix A.

Theorem 2. The MPC protocol given in Appendix $A$ evaluates a circuit with $c_{I}$ input, $c_{R}$ random, $c_{M}$ multiplication, and $c_{O}$ output gates, with communicating $\mathcal{O}\left(\left(c_{I} n+c_{R} n+c_{M} n+c_{O} n+D_{M} n^{2}\right) \kappa+n \mathcal{B} \mathcal{A}(\kappa)\right)$ bits, which amounts to $\mathcal{O}\left(\left(c_{I} n+\right.\right.$ $\left.\left.c_{R} n+c_{M} n+c_{O} n+D_{M} n^{2}\right) \kappa+n^{3} \kappa\right)$ bits, where $D_{M}$ denotes the multiplicative depth of the circuit. The protocol is perfectly secure against an active adversary corrupting $t</ n / 3$ players.

\section{Conclusions}

We have presented a perfectly secure multi-party computation protocol with optimal security $(t<n / 3)$, which communicates only $\mathcal{O}(n)$ field elements per multiplication.

Compared with the previously most efficient perfectly-secure MPC protocol [HMP00, this is a speedup of $\theta\left(n^{2}\right)$ with the same level of security.

Compared with the previously "most secure" MPC protocol with linear communication complexity DN07, this improves the security from unconditional to perfect, and at the same time slightly improves the communication overhead (from $\mathcal{O}\left(n^{4} \kappa\right)$ in DN07 to $\mathcal{O}\left(n^{3} \kappa\right)$ here).

This speed-up was possible due to a new technique, so-called hyper-invertible matrices. Such matrices allow to detectably generate $\Omega(n)$ random sharings at costs $\mathcal{O}\left(n^{2}\right)$, with perfect security (i.e., without any probabilistic checks as used in all previous highly-efficient MPC protocols). We believe that this approach is much more natural than the previous approach with probabilistic checks (for example, DN07 needs to work in an extension field to keep the error-probability small).

\section{References}

[Bea91a] Beaver, D.: Efficient multiparty protocols using circuit randomization. In: Feigenbaum, J. (ed.) CRYPTO 1991. LNCS, vol. 576, pp. 420-432. Springer, Heidelberg (1992)

[Bea91b] Beaver, D.: Secure multiparty protocols and zero-knowledge proof systems tolerating a faulty minority. Journal of Cryptology, 75-122 (1991) 
[BGP92] Berman, P., Garay, J.A., Perry, K.J.: Bit optimal distributed consensus. In: Computer Science Research, Preliminary version has appeared in Proc. 21st STOC, pp. 313-322 (1992)

[BGW88] Ben-Or, M., Goldwasser, S., Wigderson, A.: Completeness theorems for non-cryptographic fault-tolerant distributed computation. In: Proc. 20th ACM Symposium on the Theory of Computing (STOC), pp. 1-10 (1988)

[BH06] Beerliova-Trubiniova, Z., Hirt, M.: Efficient multi-party computation with dispute control. In: Halevi, S., Rabin, T. (eds.) TCC 2006. LNCS, vol. 3876, pp. 305-328. Springer, Heidelberg (2006)

[CCD88] Chaum, D., Crépeau, C., Damgård, I.: Multiparty unconditionally secure protocols (extended abstract). In: Proc. 20th ACM Symposium on the Theory of Computing (STOC), pp. 11-19 (1988)

[CDvdG87] Chaum, D., Damgård, I., van de Graaf, J.: Multiparty computations ensuring privacy of each party's input and correctness of the result. In: Pomerance, C. (ed.) CRYPTO 1987. LNCS, vol. 293, pp. 87-119. Springer, Heidelberg (1988)

[CW92] Coan, B.A., Welch, J.L.: Modular construction of a Byzantine agreement protocol with optimal message bit complexity. Information and Computation 97(1), 61-85 (1992); Preliminary version has appeared in Proc. 8th PODC (1989)

[DN07] Damgård, I., Nielsen, J.B.: Robust multiparty computation with linear communication complexity. In: Menezes, A. (ed.) CRYPTO 2007. LNCS, vol. 4622, Springer, Heidelberg (2007)

[GHY87] Galil, Z., Haber, S., Yung, M.: Cryptographic computation: Secure faulttolerant protocols and the public-key model. In: Pomerance, C. (ed.) CRYPTO 1987. LNCS, vol. 293, pp. 135-155. Springer, Heidelberg (1988)

[GMW87] Goldreich, O., Micali, S., Wigderson, A.: How to play any mental game - a completeness theorem for protocols with honest majority. In: Proc. 19th ACM Symposium on the Theory of Computing (STOC), pp. 218-229 (1987)

[HMP00] Hirt, M., Maurer, U., Przydatek, B.: Efficient secure multi-party computation. In: Okamoto, T. (ed.) ASIACRYPT 2000. LNCS, vol. 1976, pp. 143-161. Springer, Heidelberg (2000)

[HN06] Hirt, M., Nielsen, J.B.: Robust multiparty computation with linear communication complexity. In: Dwork, C. (ed.) CRYPTO 2006. LNCS, vol. 4117, pp. 463-482. Springer, Heidelberg (2006)

[RB89] Rabin, T., Ben-Or, M.: Verifiable secret sharing and multiparty protocols with honest majority. In: Proc. 21st ACM Symposium on the Theory of Computing (STOC), pp. 73-85 (1989)

[Sha79] Shamir, A.: How to share a secret. Communications of the ACM 22, 612-613 (1979)

[Yao82] Yao, A.C.: Protocols for secure computations. In: Proc. 23rd IEEE Symposium on the Foundations of Computer Science (FOCS), pp. 160-164. IEEE, Los Alamitos (1982) 


\section{Appendix}

\section{A Totally Linear Protocol}

To construct a totally linear MPC protocol we propose a more efficient input protocol. For the sake of simpler presentation we assume that all inputs are given at the beginning of the computation stage.

We first present the input protocol Linearlnput that allows a set of dealers $D \subset \mathcal{P} \cup \mathcal{U}$ each having $T$ inputs to (robustly) share these inputs among the players in $\mathcal{P}^{\prime}$ (using pre-computed $t$-sharings of random values). If there is a user with more than $T$ inputs, he plays a role of more dealers.

Protocol Linearlnput (every $D_{k} \in D$ having inputs $s_{k}^{(1)}, \ldots, s_{k}^{(T)}$ with associated random

$$
\left.t \text {-sharings }\left[r_{k}^{(1)}\right]_{t}, \ldots,\left[r_{k}^{(T)}\right]_{t}\right)
$$

1. Reconstruct: For every $D_{k} \in D$ and every $l=1, \ldots, T$ invoke $\operatorname{Recons} \operatorname{Priv}\left(D_{k},\left[r_{k}^{(l)}\right]_{t}\right)$ to reconstruct the secret random value $r_{k}^{(l)}$ towards $D_{k}$.

2. Compute Difference: Every $D_{k} \in D$ computes for every $l=1, \ldots, T$ the difference $d_{k}^{(l)}=s_{k}^{(l)}-r_{k}^{(l)}$.

3. BRoAdCAST: Invoke Broadcast to let every dealer $D_{k} \in D$ broadcast (towards the players in $\left.\mathcal{P}^{\prime}\right)$ the $T$ computed differences $d_{k}^{(1)}, \ldots, d_{k}^{(T)}$.

4. Compute Locally and Output: For every $D_{k} \in D$ and every $l=1, \ldots, T$ the players in $\mathcal{P}^{\prime}$ (locally) compute the sharing of the input $s_{k}^{(l)}$ as $\left[s_{k}^{(l)}\right]_{t}=$ $\left[d_{k}^{(l)}\right]_{0}+\left[r_{k}^{(l)}\right]_{t}$.

The robust protocol Broadcast is constructed in three steps.

We first present a non-robust broadcast protocol for $\mathcal{P}^{\prime} \mathrm{PE}-$ Broadcast.

Note, that broadcasting a value can be interpreted as sharing this value with degree zero, thus checking whether every player distributed his value consistently is the same as checking the correctness of sharings with degree zero, which we can easily do applying HIM.

Protocol PE - Broadcast (every $P_{i} \in \mathcal{P}^{\prime}$ has input $x_{i}$ )

1. Distribute Values: Every $P_{i}$ shares his input with Share $\left(P_{i}, x_{i}, d=0\right)$, i.e. sends $x_{i}$ to every $P_{j} \in \mathcal{P}^{\prime}$. Resulting in $n^{\prime}$ (supposed) 0-sharings

$$
\left[x_{1}\right]_{0}, \ldots,\left[x_{n^{\prime}}\right]_{0}
$$

2. Apply HIM $M$ : The players in $\mathcal{P}^{\prime}$ compute locally the 0 -sharings $\left[\widehat{x}_{1}\right]_{0}, \ldots,\left[\widehat{x}_{n^{\prime}}\right]_{0}$ as

$$
\left(\left[\widehat{x}_{1}\right]_{0}, \ldots,\left[\widehat{x}_{n^{\prime}}\right]_{0}\right)=M\left(\left[x_{1}\right]_{0}, \ldots,\left[x_{n^{\prime}}\right]_{0}\right)
$$

3. CHECK: Every $P_{i} \in \mathcal{P}^{\prime}$ checks the correctness of $\left[\widehat{x}_{i}\right]_{0}$. For this every $P_{j} \in \mathcal{P}^{\prime}$ sends his share of $\widehat{x}_{i}$ to $P_{i}$. If the values received by $P_{i}$ are not 0 -consistent (equal), $P_{i}$ gets unhappy. 
4. Output: Every $P_{j} \in \mathcal{P}^{\prime}$ outputs the values received in Step 1.)

Now we construct a robust broadcast protocol for $\mathcal{P}^{\prime}$ BroadcastFor $\mathcal{P}^{\prime}$ using $P E-$ Broadcast, player elimination and segmentation. BroadcastFor $\mathcal{P}^{\prime}$ allows the players in $\mathcal{P}^{\prime}$, each holding $\ell$ values $x_{i}^{(1)}, \ldots, x_{i}^{(\ell)}$ to broadcast this values among the players in $\mathcal{P}^{\prime}$.

\section{Protocol BroadcastFor $\mathcal{P}^{\prime}$}

For each segment $k=1, \ldots, t$ (of length $\ell^{\prime}=\left\lceil\frac{\ell}{t}\right\rceil$ ) do:

0 . Every $P_{i} \in \mathcal{P}^{\prime}$ sets his happy-bit to happy.

1. PE-Broadcast: Invoke PE - Broadcast $\ell^{\prime}=\left\lceil\frac{\ell}{t}\right\rceil$ times in parallel, i.e. for $l=1, \ldots, \ell^{\prime}$ invoke PE - Broadcast to let every $P_{i} \in \mathcal{P}^{\prime}$ broadcast his input $x_{i}=x_{i}^{\left(l+(k-1) \ell^{\prime}\right)}$.

2. Fault Detection: Reach agreement whether or not at least one player is unhappy:

2.1 Every $P_{i} \in \mathcal{P}^{\prime}$ sends his happy-bit to every $P_{j} \in \mathcal{P}^{\prime}$, who gets unhappy if at least one $P_{i}$ claims to be unhappy.

2.2 The players in $\mathcal{P}^{\prime}$ run a consensus protocol on their respective happybits. If the consensus outputs "happy", then the generated triples are outputted and the segment is finished. Otherwise, the following FaultLocalization step is executed.

3. Fault Localization: Localize $E \subseteq \mathcal{P}^{\prime}$ with $|E|=2$ and at least one player in $E$ being corrupted:

3.0 Denote the player $P_{r} \in \mathcal{P}^{\prime}$ with the smallest index $r$ as the referee 7

3.1 Every $P_{i} \in \mathcal{P}^{\prime}$ sends everything he received and all random values he chose during the computation of the actual segment (including fault detection) to $P_{r}$.

3.2 Given the values received in Step 3.1, $P_{r}$ can reproduce every message that should have been sent (by applying the respective protocol instructions of the sender), and compare it with the value that the recipient claims to have received. Then $P_{r}$ broadcasts $\left(l, i, j, x, x^{\prime}\right)$, where $l$ is the index of a message where $P_{i}$ should have sent $x$ to $P_{j}$, but $P_{j}$ claims to have received $x^{\prime} \neq x$.

3.3 The accused players broadcast whether they agree with $P_{r}$. If $P_{i}$ disagrees, set $E=\left\{P_{r}, P_{i}\right\}$, if $P_{j}$ disagrees, set $E=\left\{P_{r}, P_{j}\right\}$, otherwise set $E=\left\{P_{i}, P_{j}\right\}$.

4. Player Elimination: Set $\mathcal{P}^{\prime} \leftarrow \mathcal{P}^{\prime} \backslash E, n^{\prime} \leftarrow n^{\prime}-2, t^{\prime} \leftarrow t^{\prime}-1$, and repeat the segment.

Finally we present the protocol Broadcast that enables a set of dealers $D$ (players or users), each holding $T$ values to robustly broadcast this values, among the players in $\mathcal{P}^{\prime}$.

7 The communication can be balanced by selecting a player who has not yet been referee in a previous segment. 
The idea of the protocol is to let every dealer expand his $T$ values to $n^{\prime}$ values (using an error-correcting code tolerating $t^{\prime}$ errors) and to send each of these values to one player in $\mathcal{P}^{\prime}$. Then the players in $\mathcal{P}^{\prime}$ invoke BroadcastFor $\mathcal{P}^{\prime}$ to broadcast the received values and final (locally) compute the original values from the broadcasted values using error-correction.

\section{Protocol Broadcast(every dealer $D_{k}$ holding $a_{k}^{(0)}, \ldots, a_{k}^{(T-1)}$ )}

1. Expand And Distribute: For every dealer $D_{k}$ denote the polynomial defined by the values $a_{k}^{(0)}, \ldots, a_{k}^{(T-1)}$ as $p_{k}(x)$, i.e.

$$
p_{k}(x)=a_{k}^{(0)}+a_{k}^{(1)} x+\ldots+a_{k}^{(T-1)} x^{T-1}
$$

- The dealer $D_{k}$ computes for every player $P_{i} \in \mathcal{P}^{\prime}$ the point $p_{k}\left(\alpha_{i}\right)$ and sends it to $P_{i}$.

2. Broadcast: The players in $\mathcal{P}^{\prime}$ invoke BroadcastFor $\mathcal{P}^{\prime}$ with $P_{i}$ having input $p_{1}\left(\alpha_{i}\right), \ldots, p_{|D|}\left(\alpha_{i}\right)$.

3. Compute and Output: For every dealer $D_{k}$ every $P_{i} \in \mathcal{P}^{\prime}$ locally computes the values $a_{k}^{(0)}, \ldots, a_{k}^{(T-1)}$ from the broadcasted values $p_{k}\left(\alpha_{1}\right), \ldots, p_{k}\left(\alpha_{n^{\prime}}\right)$ (using error-correction). 